American Journal of Environmental Sciences 5 (4): 562-569, 2009

ISSN 1553-345X

(C) 2009 Science Publications

\title{
Integration of Electrical and Electromagnetic Investigation for Contaminated Site
}

\author{
${ }^{1}$ Alberto Godio and ${ }^{2}$ Mario Naldi \\ ${ }^{1}$ DITAG-Department of Land, Environment and Geo-Engineering, \\ Politecnico di Torino, 10129 Turin, Italy \\ ${ }^{2}$ Techgea Sas, Turin, Italy
}

\begin{abstract}
Problem statement: The environmental assessment of industrial areas is required to minimize the risk of soil and groundwater contamination. The survey of wide areas (e.g., some hectares) provides a high economical and complex logistical impact when random strategies of soil sampling are adopted. Recently, geophysical methods had been successfully adopted to drive the process of the spatial sampling of the soil. Approach: The main goal was to verify the reliability of the geophysics for the detection of buried wastes in an industrial area previously involved in the vehicle demolition. Two geophysical methods were applied (1) the electromagnetic frequency domain, using a multi-frequency broadband system with a fixed spacing between the transmitter and receiver coils $(2 \mathrm{~m})$ to observe the soil electrical conductivity and in-phase response (2) the electrical resistivity and chargeability tomographies, performed along several transects. We applied the Kriging method to map the conductivity and the in-phase response. Standard inversion procedure was adopted to interpret the resistivity and chargeability data. Results: We found a very similar response of the electromagnetic survey in range between 2000 and 40,000 Hz: The investigation depth (3-4 m) was mainly related to the coil spacing. The survey pointed out some anomalies caused by bio-geochemical activity in the active waste deposits. The resistivity and chargeability sections confirmed that the electromagnetic anomalies were related to the waste. The high chargeability values (up to $100 \mathrm{mV} / \mathrm{V}$ ) were associated to the leakages of metals of the wastes. Conclusion: The electromagnetic survey, even if sensitive to the presence of cultural noise (fence, metallic object on the ground), permitted to detect the industrial waste; the electrical resistivity and chargeability data pointed out the main contaminated zones with good accuracy. The further development may focus on the relationship between the nature of waste and the geophysical response.
\end{abstract}

Key words: Electrical and electromagnetic methods, industrial waste

\section{INTRODUCTION}

The preliminary assessment and the remedial design of contaminated sites require to acquire information about the contaminant, the geology and the hydrogeology of the site. The main objectives are to improve the knowledge of the hydro-geological setting of the area, to detect the macro area potentially contaminated, to detect waste disposal.

The integration of cost-effective geophysical investigation at large scale with accurate geochemical analysis of samples of the subsoil and groundwater is recommended in order to quantitatively estimate the extent and the level of the contamination. Traditionally techniques (such as soil sampling, core drillings) are invasive, time consuming, expensive; and do not give a continuous image of the subsurface. Geophysical methods such as Ground Penetrating Radar (GPR), Frequency Domain Soundings (FDEM), Electrical Resistivity (ER and ERT) and Self-Potential (SP) are methods for characterizing subsurface properties and spatial structure ${ }^{[1-4]}$. The non-invasive geophysical methods map zones or layers of different physical characteristics. Conventional geophysical imaging involving data responding to one physical property of the ground suffers from inadequate model resolution in complex sites. Joint analysis, combining measurements from multiple geophysical and/or hydrochemical measurements is a better approach and leads to accurate characterization of soil spatial variability ${ }^{[5,6]}$.

The study deals with the description of a geophysical survey on a contaminated site to analyze

Corresponding Author: Alberto Godio, DITAG-Department of Land, Environment and Geo-Engineering, Politecnico di Torino, C.So Duca Degli Abruzzi, 24, Torino, I-10129, Italy Tel: + 390115647656 Fax: + 390115647691 
the efficiency and reliability of the investigation for the design of subsequent geochemical characterization.

The aim of the geophysical investigation was to delineate the spatial extension of the contaminated area and estimating the maximum depth of the waste deposits. The approach combines electromagnetic frequency domain investigation and Electrical Resistivity Tomography (ERT) to map the extension of the waste material and evaluate the depth reached by the contamination. A great care in data processing must be adopted to evaluate the sensitivity of the methods in order to use the geophysical results for a preliminary estimate of the contaminated volume.

The mapping by electromagnetic Frequency Domain (FDEM) method in the frequency range between 2 up to $40 \mathrm{kHz}$ permitted a preliminary analysis of the main hot spots related to the anthropogenic activities.

We applied the 2D electrical resistivity and chargeability tomography to map the vertical extension of the waste deposal in the site interested by a complex contamination due to automotive activity with presence in the subsoil of metallic refuse and fluids due to spill of oils by the buried vehicles.

The results of the geophysical survey have driven the subsequent phase of the clean up planning of the area with a reduction of the cost of the direct investigation by boreholes.

\section{MATERIALS AND METHODS}

Site description: The site is characterized by ancient alluvial deposits, mainly including gravel and sandy materials. The sediments with an average thickness of about 20-25 m overlie a metamorphic bedrock. The first 2-3 $\mathrm{m}$ are compounded by man-made debris, potentially contaminated or hosted the waste disposal (Fig. 1). The near surface aquifer hosted in alluvial materials is not contaminated, according to the Italian rules.

The area, partially covered by concrete, served as waste disposal of materials, that derived from automotive demolition activities. At the beginning the industrial site hosted the waste materials that derived from automotive industry; during the industrial activity, the site has been gradually transformed in a not controlled landfill of different types of wastes, derived from demolition of end of life vehicles, electrical household appliances, tires and other wastes with high content of metals. The stakeholders of the demolition plant put down directly on the ground or on the asphalt of the plant the rests of the end of life vehicles, with batteries, plastic materials and metallic wastes, gasoline and oils and other fluids of the vehicles.

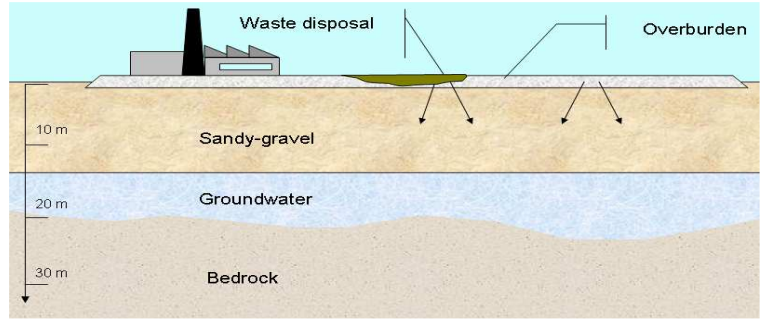

Fig. 1: Sketch of the geological and conceptual model of the site

The residual part of the demolition of end of life vehicles cannot be easily classified from the chemical point o view; the waste is characterized by the plastic and grubber, fluff, metallic (non ferrous) materials, glass and in minor part wood are recognized. The heavy metals such as lead, zinc and copper and oil fluids are residuals of inadequate operations of the preliminary phase of clean up of the vehicles.

The material appears heterogeneous both in size and composition; the intrinsic heterogeneities provide a complexity in the analytical determination on the representative samples of the waste materials, with a large spread of the results deriving from different samples.

The waste directly posed on the ground surface determines a diffuse contaminations mainly due to the leaching of the hydrocarbons (gasoline and diesel oil, oils and PCB) and of heavy metals (cadmium, lead, zinc, copper). Fortunately, the monitoring of the groundwater quality within the control wells, located hydraulically upstream and downstream, doesn't point out any evidence of the water contamination; this indicates that the environmental impact is limited to the soil only.

Because the waste deposal was completely random on a wide area, approximately of $200 \times 100 \mathrm{~m}$, the planning of the trenches and the positioning of the boreholes for the soil sampling was a complex challenge; taking into account this complexity the geophysical survey was focused to point out the main hot spots that could be related to the waste disposal.

Electromagnetic properties of soil: Electrical and electromagnetic investigation for contaminated site is mainly based on the spatial and temporal observation of the electromagnetic intrinsic properties of the subsoil with different methodological approach (galvanic contact or induction) and frequency of the applied electromagnetic field. The electro-magnetic properties of the materials are the bulk conductivity which drives the electrical conduction of current in the medium; the 
inverse of the conductivity is the electrical resistivity; the polarizability controls the capability of the medium to accumulate and release the electrical energy; the magnetic susceptibility governs the magnetic response of the medium.

In water bearing materials the current conduction is due to the electrolytic conduction, where the electrolyte conductivity of the pore water has the dominant influence upon the matrix conductivity. Moreover, the contaminants (miscible or not miscible) provide for complex chemical and physical interactions between fluid and solid matrix.

In a porous aquifer, the electrical conductivity depends on the porosity of the medium, water saturation, total dissolved solids, ionic mobility, temperature. In fractured rocks, the electrical conductivity depends on the density of fractures; tortuosity of the electrical paths inside the fracture. In the porous medium with clay particles, the electrical conductivity is affected by the specific (surface) conductivity of the clay material. Moreover effects of biological activity on organic matter provides for an increase of the minerals dissolved in fluid. The biochemical activity also modifies the magnetic properties of waste or contaminated soil changing the susceptibility contrast between the top-soil and waste material.

Effect of contaminant: The behavior of the electrical resistivity of contaminants, with respect to the host environment, depends on several factors, such as the host lithology, the soil moisture and the solubility of the contaminants in the groundwater. For organic contaminants that are not miscible with water, such as diesel oil and gasoline, the complexity of chemicalphysical reactions with the host environment makes it unrealistic to determine a typical range of resistivity which could enable differentiation of the plume from its surroundings. These phenomena can significantly affect the electrical and electromagnetic properties of the soil and groundwater and make the clear and unambiguous detection of contaminants very difficult. Many published papers have reported seemingly contradictory results on the electrical and electromagnetic response of different contamination events. Mazac et al. ${ }^{[7]}$ pointed out the shortcomings of surface electrical methods in the detection of thin layers of the free hydrocarbon phase above the water table, although borehole investigations and tomographic data processing can overcome this problem in some favorable conditions $^{[8-10]}$

The electromagnetic response at radiofrequency is complicated by the possible effects of the gas and by the different behavior of the contamination in the vadose zone and underlying groundwater zone ${ }^{[11]}$ Geophysical methods are unable to detect some contaminants at the low concentrations of parts per billion that are of regulatory concern, although toluene can sometimes be detected with the complex resistivity technique due to of its chemical reactions with clay minerals. Complex electrical tomography has been reported to be successful in the detection of diesel pollution in the vadose zone ${ }^{[12]}$, even though great care must be taken to avoid electromagnetic coupling effects on the experimental data.

Methods: Several methods, stand-alone and in a joint approach, are usually adopted in environmental characterization. The Electrical Tomography (ET) and low Frequency Electromagnetic (FEM) methods are the most diffused; these methods permit to survey wide areas at low costs and reduced time effort.

The Electrical Resistivity Tomography (ERT) permits to detect and image variations in the resistivity of sub-soil. The electrical tomography is based on the automated measurement and computerized analysis of electrical resistivity change. Because the bulk resistivity of rock and soil depends on the water content, chemistry and temperature, electrical tomography is useful for imaging subsurface processes involving changes in water saturation or water chemistry.

The electromagnetic method in frequency domain allows one to measure the response of the subsoil and of buried objects in terms of the secondary magnetic field at the selected frequencies. As a rule of thumb, low frequencies mean higher depths of investigation with a lack in spatial resolution compared to the high frequencies.

Apparatus: Electrical measurements were acquired using a Syscal instrument connected to lines of 72 electrodes. The Syscal R1 (IRIS Instrument) for DC electrical tomography with a maximum voltage of $600 \mathrm{~V}$ and a maximum current up to $1.2 \mathrm{~A}$ was utilized. Accurate measurements were carried out with high power instrument in order to obtain reliable information at deep up to $15-20 \mathrm{~m}$. All measurements have been carried out using the Wenner/Schlumberger array with 2 or $3 \mathrm{~m}$ of electrodes spacing to obtain a good compromise between high stability of the measure and satisfactory spatial resolution. Moreover the digital stacking of the received signal provides a high signal to noise ratio and good quality data with very low percentage of rejected measurements. The adopted array is relatively sensitive to vertical changes of resistivity below the centre of the array, with low 
sensitive in delineating narrow vertical structures. On the other hand, good quality data can be collected if compared with other conventional arrays in noisily areas. In such a context, the choice of the array configuration was conditioned by the compromise between spatial resolution and the need to acquire good quality data in an area with potentially high background noise due to the presence of the resistive coverage and man-made structures.

The electromagnetic ground conductivity meter is compound by a transmitter and receiver coil, that are separated by a short distance (less than $2 \mathrm{~m}$ ). The operating frequency is low enough at the intercoil spacing that the electrical skin depth is always significantly greater than the intercoil spacing. Under this condition, technically known as operation at low induction number, the quadrature phase component (out of phase with respect to the primary field) of the received signal, for low and moderate conductivities, can be related to the apparent electrical conductivity of the soil. Details on the basic principles of the technique and the equipment are well documented ${ }^{[13]}$.

The device permitted to investigate the electromagnetic response of the subsoil (in-phase and in quadrature component) at several frequencies in a few seconds for each station; a $2 \mathrm{~m}$ square grid has been used for the acquisition.

Data acquisition: A preliminary survey of the area was carried out by the Electromagnetic Multifrequency (EM) method in the frequency range between 2000 and $40000 \mathrm{~Hz}$ : The multifrequency acquisition is suitable to map the electromagnetic response of the subsoil up to few meters in depth from the surface. The maximum depth of investigation depends on several factors, that are related to instrumental parameters (frequency, intercoil distance), to the acquisition procedure (coil orientation) and finally to the electromagnetic behavior of the subsoil and the contrast of electromagnetic parameters of the target with respect to the host environment.

In the selected area, the EM mapping was performed with a grid of $2 \times 2 \mathrm{~m}$ in a broad-line approach (transmitter and receiver perpendicular to the profiles) in vertical dipole configuration (co-planar coils in a horizontal plane).

The in-phase and quadrature values of the secondary magnetic field are normalized to the primary magnetic field and expressed in parts per million (ppm). In many applications the mapping of the inphase and quadrature data is sufficient to localize the targets and estimate the shape and size of the buried objects. In other cases, a more quantitative interpretation is necessary for a more exhaustive site characterization.

We performed 15 Electrical Resistivity and Polarizability Tomography (ERT), using 72 electrodes. The location of the ERT profiles were planned in order to cross the main suspected area as previously indicated by the EM mapping, taking the available space inside the industrial site into account.

The line 1 was realized outside the expected contaminated area for calibrating the background response of the not contaminated soil. The corresponding vertical sections of resistivity and chargeability data were used as reference for the interpretation of the remaining lines.

Analysis: The processing of electromagnetic data involved the following steps:

- Mapping of electromagnetic in-phase and quadrature and the electrical conductivity response at the selected frequencies

- Forward modeling of the electromagnetic response using simple models, which simulated the main anomalies due to the waste deposits

- Elaboration of 2D pseudo-sections by interpolating the results of many soundings along each profile

- The 3D rendering permitted to delineate the main anomalies potentially related to the waste deposits

The effective depth of investigation was estimated according to the cumulative response of the electromagnetic device, operating at low induction number; the induction number is the ratio between the intercoil spacing (s) and the skin depth (d). A rough estimate of maximum depth of investigation is based on the skin depth, which is a conventional depth from the source at which the amplitude of the electromagnetic field is attenuated by 1/e (Euler number). Anyway, at a fixed intercoil distance for a low induction system the depth-sensitivity function is independent from the frequency and the electrical soil conductivity; the sensitivity depends on the intercoil distance ${ }^{[14]}$. For a horizontal coplanar system, we consider that the method is sensitive to a depth less than $2 \mathrm{~m}$ (equal to the coil spacing).

The 2D mapping of the area at different frequencies is useful to analyze the behavior of different waste material; it can be considered that using a multifrequency broadband device with a fixed intercoil spacing, the electromagnetic response generates by a buried target is characterized by a conductive behavior at low frequency and the inductive 
behavior at higher frequency. This assumption guides the qualitative interpretation in relating the geophysical anomalies to the waste material; the ferrous waste can be more easily detected observing the high frequency response: On the other hand leakage and percolation of saline contaminant dispersed in fluid phase could be better related to the electromagnetic response at low frequency.

The quantitative interpretation of the electromagnetic data involves different approaches, that can be useful for a fast and rough estimation of the shape and depth of the electrically conductive anomalies. Wesley et $a l^{[15]}$ and West et al. ${ }^{[16]}$ determined the response of a perfectly conducting half plane. The response of small systems to simple 3D targets can be obtained using numerical solutions ${ }^{[17]}$. The electromagnetic solution for a magnetic dipole loop source and a receiver for a n-layered earth was studied by Anderson ${ }^{[18]}$. according to the original works by ${ }^{[19,20]}$ for homogeneous soils.

A rough interpretation can be performed by the comparison of the experimental data with the shape and amplitude of the theoretical response due to simple models ${ }^{[21]}$. We performed the modeling of the EM response at different frequencies for a conductive and permeable sphere embedded in a more resistive and not-magnetizable soil. An example is plotted in Fig. 2.
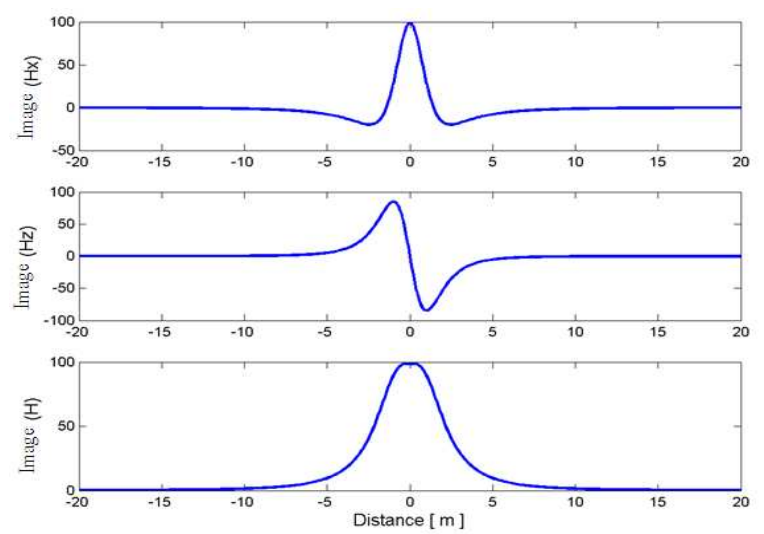

Fig. 2: Example of the theoretical electromagnetic anomaly along a profile: The imaginary part or quadrature response of the horizontal (Hx) vertical $(\mathrm{Hz})$ and resultant $(\mathrm{H})$ of the secondary magnetic field of a conducting sphere in a homogeneous time varying field, for a sphere of radius $1 \mathrm{~m}$ with a conductivity of $0.1 \mathrm{~S} \mathrm{~m}^{-1}$ : The centre of the sphere is located at the coordinates $\mathrm{x}=0 \mathrm{~m}, \mathrm{z}=-2 \mathrm{~m}$ (values are in ppm)
The main assumptions on the model parameters (size and depth) are based on the analysis of the electrical tomography results. Particularly, the electrical resistivity and polarizability tomography, performed along several transects, permitted to calibrate the electromagnetic results and furnish some details on the effective depth of the waste deposits.

The ERT images were obtained using a leastsquares inversion technique. The process selects a starting resistivity model of the subsoil; at each iteration a model parameter change vector is calculated and hence the resistivity model is modified in order to minimize the sum of square differences between the model response and the observed data. All the section was processed using the least-squares smoothnessconstrained inversion algorithm ${ }^{[22-24]}$.

\section{RESULTS}

Electromagnetic results: The Fig. 3 shows the projection on a topographic map of the electrical resistivity as mapped by the FDEM survey at frequency of $5025 \mathrm{~Hz}$. The plot points out the sensitivity of the method to the interferences due to the presence of metallic surface in the pavement (concrete with metallic rebar), as evident in the western part of the area. In the central and eastern part of the surveyed area, the electromagnetic maps depicted some conductive hot spots (Fig. 4), that are not related to near surface features.

The maps of the quadrature response at several frequencies confirmed the presence of a main hot spot (the relative coordinates $X=0-10$ and $Y=30-40 \mathrm{~m}$ in Fig. 5). This anomaly is well reproduced for all the adopted frequencies.

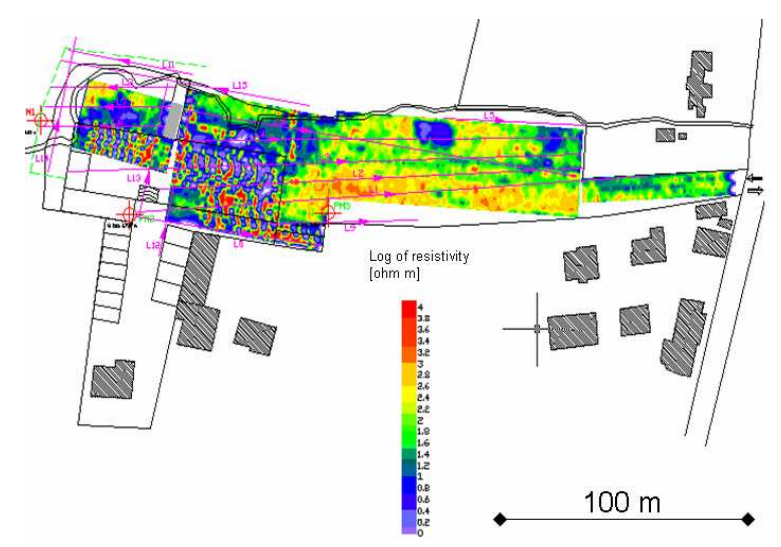

Fig. 3: Test site and the electrical conductivity map of the subsoil using the frequency domain electromagnetic method: The straight lines (magenta) indicates the electrical tomographies 


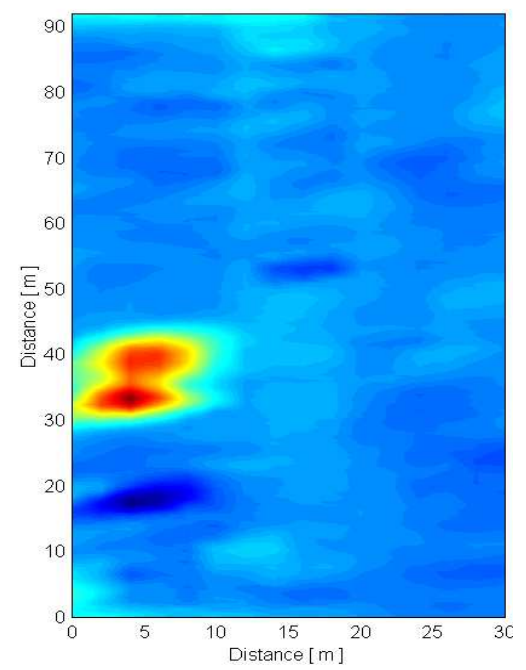

Fig. 4: Map of the electromagnetic response (quadrature) of a sub-area of the investigated site; data acquired at $5025 \mathrm{~Hz}$; the red-colored zone detects the peak of the amplitude of the electromagnetic response
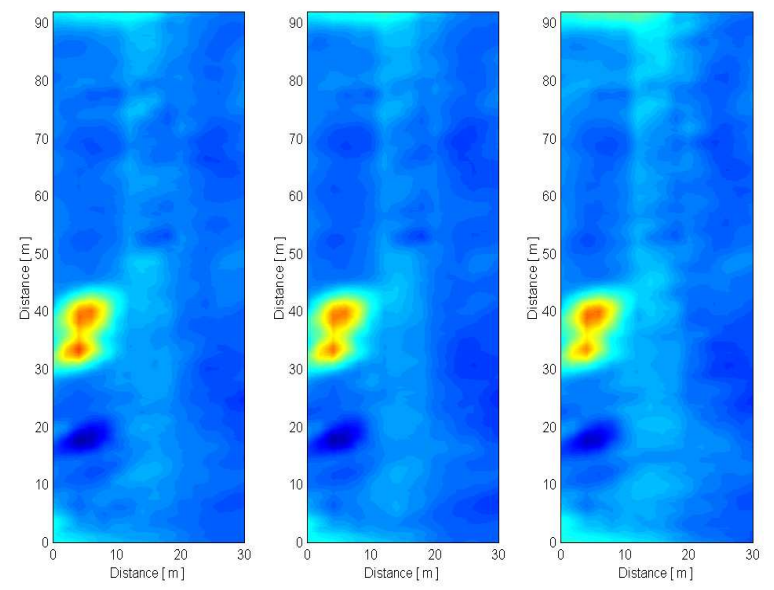

Fig. 5: Maps of the quadrature response of the same subarea of fig. 4; (Left): Data at $15025 \mathrm{~Hz}$; (Center): Data at $16875 \mathrm{~Hz}$; (Right): Data at $20025 \mathrm{~Hz}$

The $3 \mathrm{D}$ rendering of the electromagnetic mapping at different frequencies is computed starting from the horizontal slices obtained at the difference frequencies.

We conventionally considered the lower frequency at deeper level of the 3D rendering, even if no relationships between the frequency response and depth can be established, according to the theoretical statements on the depth-sensitivity of the method as previously described. The 3D image (Fig. 6) focuses on the central part of the investigated area; the image points out the presence of two main electromagnetic anomalies.

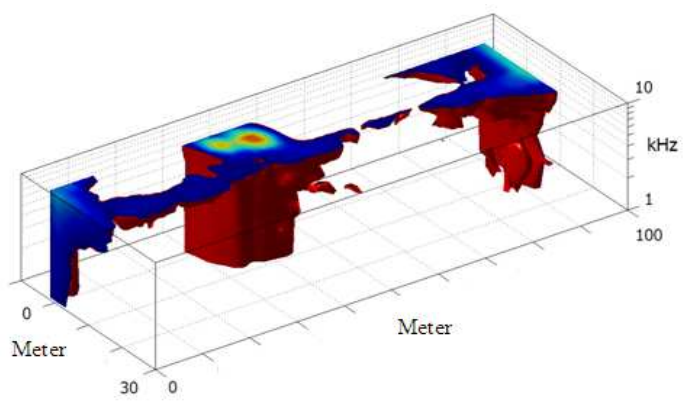

Fig. 6: 3D image of the quadrature response obtained by $3 \mathrm{D}$ interpolation of horizontal slice

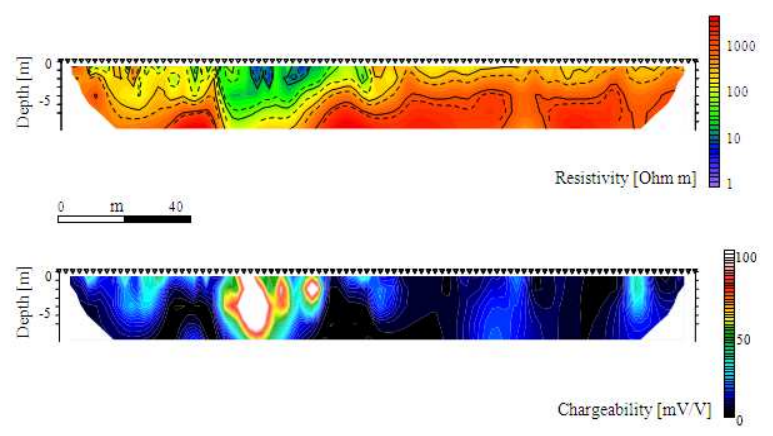

Fig. 7: Line 3-top electrical resistivity tomography, bottom) chargeability tomography; the high values of conductivity correspond to anomaly of chargeability and pointed out the waste disposal.

Electrical tomography: The Fig. 7 shows the vertical models of electrical resistivity and chargeability of the line n.3; the results pointed out a resistivity model with a homogeneous high resistive background (above $1000 \mathrm{ohm} \mathrm{m}$ ). The main hot spots (high conductive zones) are well depicted at the coordinates between 50 and $70 \mathrm{~m}$.

The vertical section of the chargeability model shows, in a background of low values of chargeability (less than 2-3 mV/V), a high level of chargeability which correspond to the same position of the main high conductive anomaly. The hot spot is characterized by resistivity values of about $100 \mathrm{ohm}$ m and chargeability up to $100 \mathrm{mV} / \mathrm{V}$. The vertical extension of the anomaly is up to 5-6 $\mathrm{m}$ from the ground. The spatial coincidence of the high conductive and chargeability anomaly makes sense to the assumption of the presence of waste material with a complex contamination still active. The subsequent direct investigation revealed the presence of mainly ferrous metallic object ands high contents of heavy metals in the surrounding subsoil. 


\section{DISCUSSION}

The presence of a near surface aquifer needs for a reclamation of the area in order to avoid migration of contaminants far away from the sources; in such a context the geophysical survey permitted a preliminary evaluation of the extension of the contaminated zone for planning the reclamation activity and estimate the entity of the claims.

The quadrature response of the electromagnetic mapping should be more sensitive to the anomalies of the electrical conductivity of the soil, while the in-phase response could be better related to the magnetic properties of the sub-soil. The simultaneous presence of high values of quadrature and in-phase components suggest that the waste is compound by ferrous material (magnetisable) with leakage effects that provided an increase of the electrical conductivity. This explains the high values of electrical conductivity as pointed out by the electrical tomography. The anomalies of chargeability should be related to the presence of intense geochemical interaction between the solid skeleton and the moisture highly enriched in metallic ions due to the leakages of the metallic waste.

The ERT method appears very sensitive to the presence of rebar in the concrete. The rebar provides for strong conductive ghosts in the ERT images: This limits the penetration depth of the survey in the area covered by the concrete. A better accuracy was obtained in are without no asphalt and concrete coverage.

The methods are suitable to be applied in alluvium deposits where the lateral changes of resistivity is not associated to variations of facies and lithologies. The preliminary evaluation of the reference parameters on the not-contaminated sandy deposits is fundamental in order to detect the heterogeneities caused by the near surface variations and to evaluate the effects on the geophysical measurements of the soil moisture.

Advantages and limits: The EM offers a very fast mapping of the electromagnetic properties of the subsoil; the use of a fixed-spacing device didn't permit to investigate a depth greater than 1.5-2 times the separation distance. Therefore, while a good lateral resolution was achieved (in order of meter or higher), the method suffers of a lack of sensitivity with depth. The use of different frequencies in a broadband range didn't provide any substantial benefit in the vertical resolution and in depth of investigation.

The main shortcomings of the electromagnetic frequency domain methods using portable transmitterreceiver devices are related to the lack in the depth resolving power and the effect of the man-made structures. The intrinsic lack in depth resolution using multi-frequency system provides a main sensitivity to the near surface features. In the application of frequency domain electromagnetic method in industrial areas, the presence of near surface metallic objects could mask the response of the deeper waste materials. While the electromagnetic response is limited to a depth of few meters, the electrical tomography is able to investigate at deeper level even if the intrinsic spatial resolution tends to degrade with depth. A more robust data interpretation was obtained by combining the results of the electrical resistivity and chargeability: the simultaneous evidence of low conductivity and high chargeability anomalies was considered as an robust evidence of the waste deposits.

\section{CONCLUSION}

The interpretation of the EM permitted to delineate the presence of concrete slabs with rebar and detect two main areas suspected to be used as waste disposal. The excavation of trenches confirmed the presence of complex contamination in the selected areas.

The analysis of geophysical data demonstrated the reliability of the 2D and 3D rendering of the frequency domain electromagnetic data to describe the response of the electromagnetic properties of the shallow subsoil (up 2-3 $\mathrm{m}$ in depth) interested by the presence of waste material. We stressed the importance to validate the electromagnetic data (depth and spatial resolution) by other geophysical methods and the hydro-geological information in order to calibrate the geophysical response and reducing the ambiguities of data interpretation. In the described examples the geophysical survey permitted to successfully detected two main areas where the industrial waste was previously buried.

\section{REFERENCES}

1. Regli, C., P. Huggenberger and M. Rauber, 2002. Interpretation of drill core and georadar data of coarse gravel deposits. J. Hydrol., 255: 234-252. http://cat.inist.fr/?aModele=afficheN\&cpsidt=13398407

2. Hubbard, S.S. and Y. Rubin, 2000. Hydrogeological parameter estimation using geophysical data: A review of selected techniques. J. Contaminant Hydrology, 45: 3-34. http://cat.inist.fr/?aModele $=$ afficheN\&cpsidt $=1471089$

3. Kowalsky, M.B., P. Dietrich, G. Teutsch and Y. Rubin, 2001. Forward modeling of groundpenetrating radar data using digitized outcrop images and multiple scenarios of water saturation. Water Res. Res., 6: 1615-1625. http://wwwesd.lbl.gov/ESD_staff/kowalsky/publications/2001 WR900015.pdf 
4. Rea, J. and R. Knight, 1998. Geostatistical analysis of ground-penetrating radar data: A means of describing spatial variation in the subsurface. Water Resources Res., 34: 329-339. http://cat.inist.fr/?aModele $=$ afficheN\&cpsidt $=1036$ 9693

5. Gallardo, L.A. and M.A. Meju, 2003. Characterisation of heterogeneous near-surface materials by joint 2D inversion of dc resistivity and seismic data. Geophys. Res. Letts., 30: 1658-1661. http://www.agu.org/pubs/crossref/2003/2003GL01 7370.shtml

6. Linde, N., A. Binley, Tryggvason, L. Pedersen and A. Revil, 2006. Improved hydrogeophysical characterisation using joint inversion of cross-hole electrical resistance and ground-penetrating radar traveltime data. Water Res. Res., 42: 12404-12404. http://cat.inist.fr/?aModele $=$ afficheN\&cpsidt $=18476182$

7. Mazac, O., L. Benes, I. Landa and A. Maskova, 1990. Determination of the extent of oil contamination in groundwater by geoelectrical methods. Geotech. Environ. Geophys. Environ. Groundwater, 2: 107-112.

8. Pidlisecky A., E. Haber E. and R. Knight, 2007. RESINVM3D: A 3D resistivity inversion package. Geophysics, 72: H1-H10. DOI: 10.1190/1.2402499

9. Daily W. and A. Ramirez, 2004. Electrical Impedance Tomography for Detection of DNAPL Contamination. Proceeding of the Symposium on the Application of Geophysics to Engineering and Environmental Problems, (SAGEEP'04), EEGS, pp: 449-454. DOI: 10.4133/1.2923357

10. Godio, A. and M. Naldi, 2003. Two-dimensional electrical imaging for detection of hydrocarbon contaminants. Near Surface Geophys., 3: 131-137. http://www.techgea.eu/articoli\%20pubblicati/Twodimensional\%20electrical\%20imaging $\% 20$ for $\% 20$ detection.pdf

11. Benson, A.K., 1995. Applications of ground penetrating radar in assessing some geological hazards: Examples of groundwater contamination, faults, cavities. J. Applied Geophys., 33: 177-193. http://cat.inist.fr/?aModele $=$ afficheN\&cpsidt $=3446$ 071

12. Kemna, A., L. Dresen and E. Raekers, 1999. Field applications of complex resistivity tomography, SEG. Expand. Abstracts, 18: 331-334. DOI: 10.1190/1.1821014

13. Won, I.J., D.A. Keiswetter, G.R.A. Fields and L.C. Sutton, 1996. GEM-2: A new multifrequency electromagnetic sensor. J. Environ. Eng. Geophys., 1: 129-137.
14. McNeill, J.D., 1996. Why doesn't Geonics Limited built a multi-frequency EM31 or EM38? Technical Note TN-30, Geonics Limited, pp: 1-6. http://www.geonics.com/pdfs/technicalnotes/tn30.pdf

15. Wesley, J.P., 1958. Response of a dyke to an oscillating dipole. Geophysics, 23: 128-133. http://adsabs.harvard.edu/abs/1958Geop...23..134 $\mathrm{W}$

16. West, G.F., 1960. Quantitative interpretation of electromagnetic prospecting measurements. Ph.D. Thesis, University of Toronto. http://adsabs.harvard.edu/abs/1960PhDT.........7W

17. Newman, G.A., G.W. Hohmann and W.L. Anderson, 1986. Transient electromagnetic response of a three-dimensional body in a layered earth. Geophysics, 51: 1608-1627. DOI: 10.1190/1.1442212

18. Anderson, W.L., 1979. Numerical integration of related Hankel transforms of orders 0 and 1 by adaptive digital filtering. Geophysics, 44: 1287-1305. http://www.whoi.edu/science/AOPE/people/achave /Site/Next_files/16.pdf

19. Wait, J.R., 1954. Mutual coupling of loops lying on the ground. Geophysics, 19: 290-296. DOI: 10.1190/1.1437996

20. Wait, J.R., 1955. Mutual electromagnetic coupling of loops over a homogeneous ground. Geophysics, 20: 630-637. DOI: 10.1190/1.1438167.

21 Wait, J.R., 1951. A conducting sphere in a time varying magnetic field. Geophysics, 16: 666-672. http://dx.doi.org/10.1190/1.1437716

22. Sasaki, Y., 1992. Resolution of resistivity tomography inferred from numerical simulation. Geophys. $\quad$ Prospect., 40: 453-463. http://cat.inist.fr/?aModele $=$ afficheN\&cpsidt $=5513$ 223

23. Degroot-Hedlin and S. Constable, 1990. Occam's inversion to generate smooth, two-dimensional models from magnetotelluric data. Geophysics, 55: 1613-1624.

http://marineemlab.ucsd.edu/resources/Pubs/OCC AM2D.pdf

24. Loke, M.H. and R.D. Barker, 1995. Least-squares deconvolution of apparent resistivity pseudosections. Geophysics, 60: 1682-1690. http://cat.inist.fr/?aModele $=$ afficheN\&cpsidt $=2903$ 763 